\title{
Exhaled Nitric Oxide Measurement may Predict Asthma Exacerbation after Stepping down Formoterol/Budesonide Combination Therapy in Adult Asthma
}

Toshihiro Shirai*, Tomotaka Kawayama, Hiroyuki Nagase, Hiromasa Inoue, Suguru Sato, Koichiro Asano and Hiroaki Kume

Early Biomarker for Treatment Study Group, Innovative Asthma Association, Japan

\begin{abstract}
Objective: The Global Initiative for Asthma (GINA) guidelines state that when asthma control is maintained for at least 3 months, treatment can be stepped down; however, prediction tools have not been established for the reappearance of symptoms and increased risk of exacerbation on stepping down treatment. This study was designed to assess whether FeNO measurement predicts asthma exacerbation after stepping down from fixed dose formoterol/budesonide combination (FBC) $9 / 320 \mu \mathrm{g}$ bid to $4.5 / 160 \mu \mathrm{g}$ bid (UMIN000005406).
\end{abstract}

Methods: Subjects included 37 patients receiving a fixed-dose FBC 9/320 $\mu$ g bid for at least 3 months, and achieving controlled asthma (GINA) in conjunction with an Asthma Control Questionnaire (5-item version (ACQ5) score $\leq 0.75$ ). Based on the FeNO value at stepping down, patients were classified into 25 patients with FeNO $<37$ $\mathrm{ppb}$ and 12 with FeNO $\geq 37 \mathrm{ppb}$. The primary endpoint was the occurrence of asthma exacerbation within 8 weeks and from 8 weeks until 12 months. Secondary endpoints, including ACQ5, FeNO, and pulmonary function tests, were measured at baseline and until 8 weeks.

Results: There was no difference in the incidence of exacerbation between patients with FeNO $\geq 37 \mathrm{ppb}$ and those with FeNO $<37 \mathrm{ppb}$ within 8 weeks; however, in a long-term follow up until 12 months, the incidence was significantly higher in patients with FeNO $\geq 37 \mathrm{ppb}$ than in those with FeNO $<37 \mathrm{ppb}$ (odds ratio $11.33,95 \%$ confidence interval 1.45 to 88.52 ). There was no statistically significant differencein changes in ACQ5, pulmonary functions, and FeNO between the 2 groups by 2-way repeated measures analysis of variance.

Conclusions: Higher FeNO levels may predict asthma exacerbation not within a short period of time, but in a long-term follow-up after stepping down FBC therapy in adult asthma.

Keywords: Asthma; Asthma control questionnaire; Exacerbation; Exhaled nitric oxide; Fixed-dose; Formoterol/budesonide combination; Step-down therapy

Abbreviations: ACQ5: Asthma Control Questionnaire: 5-item version; ACT: Asthma Control Test; ANOVA: Analysis of Variance; FBC: Formoterol/Budesonide Combination; FEF50\%: Forced Expiratory Flow at 50\% of FVC; FeNO: Fractional Exhaled Nitric Oxide; GINA: Global Initiative for Asthma; ICS: Inhaled Corticosteroid LABA: Long-Acting 32 -Agonist; Min\% Max PEF: Lowest PEF Expressed as a Percentage of the Highest PEF; PEF: Peak Expiratory Flow; ROC: Receiver-Operator Characteristic; SABA: Short-Acting $\beta 2$-Agonist; SFC: Salmeterol/Fluticasone Combination

\section{Introduction}

The current asthma guidelines emphasize achieving current control and reducing future risk [1,2]. The Global Initiative for Asthma (GINA) guidelines state that when asthma control is maintained for at least 3 months, treatment can be stepped down [1]; however, there is little information on the optimal timing, sequence, and magnitude of treatment reductions in asthma, and the approach will differ from patient to patient depending on the medications and the doses to achieve control. Prediction tools for the reappearance of symptoms and increased risk of exacerbation would be useful for stepping down treatment.

Measurement of fractional exhaled nitric oxide (FeNO) is a quantitative, noninvasive, simple, and safe method of evaluating eosinophilic airway inflammation, the hallmark of asthma [3]. Common reasons for measuring FeNO include a guide to changes in doses of anti-inflammatory medications: stepping down dosing, stepping up dosing, or discontinuation of anti-inflammatory medications [3]. Previous studies indicated that as a predictor of asthma control, FeNO is no better than more conventional tests and that the predictive values of a single measurement of FeNO for loss of asthma control are insufficiently sensitive and specific [3-5]; however, these studies were concerned with adjusting the inhaled corticosteroid (ICS) dose in not only controlled but uncontrolled patients while very few have examined the usefulness of FeNO measurement as a predictor of asthma exacerbation in patients with well-controlled asthma receiving an ICS in combination with a long-acting $\beta 2$-agonist (LABA). Gelb et al. reported that combined baseline FeNO $\geq 28 \mathrm{ppb}$ and FEV $1 \leq 76 \%$ of predicted were reliable predictors of exacerbation in patients who were clinically stable for 6 weeks and receiving salmeterol/fluticasone combination (SFC) 100/500 $\mu$ g per day [6]. Hojo et al. found that FeNO $\leq 28 \mathrm{ppb}$ plus an Asthma Control Test (ACT) score $\geq 22$ for more than 3 months was a safe criterion for stepping down formoterol/budesonide combination (FBC) $18 / 640 \mu \mathrm{g}$ per day to $9 / 320 \mu \mathrm{g}$ per day without an

*Corresponding author: Toshihiro Shirai, Department of Respiratory Medicine Shizuoka General Hospital, 4-27-1 Kita-Ando, Aoi, Shizuoka, 420-8527 Japan, Tel: 81-54-247-6111; Fax: 81-54-247-6140; E-mail: toshihiro-shirai@i.shizuoka-pho.jp

Received April 01, 2013; Accepted May 15, 2014; Published May 20, 2014

Citation: Shirai T, Kawayama T, Nagase H, Inoue H, Sato S, et al. (2014) Exhaled Nitric Oxide Measurement may Predict Asthma Exacerbation after Stepping down Formoterol/Budesonide Combination Therapy in Adult Asthma. J Allergy Ther 5 173. doi:10.4172/2155-6121.1000173

Copyright: (c) 2014 Shirai T, et al. This is an open-access article distributed under the terms of the Creative Commons Attribution License, which permits unrestricted use, distribution, and reproduction in any medium, provided the original author and source are credited. 
increased incidence of exacerbation [7]. Thus, we hypothesized that FeNO measurement would predict asthma exacerbation after stepping down FBC therapy in patients with well-controlled asthma. In this prospective, multicenter, open-label, uncontrolled observational study, we assessed the effect of stepping down treatment regimens, from FBC delivered via Turbuhaler $4.5 / 160 \mu \mathrm{g}^{2}$ inhalations bid to 1 inhalation bid, on the occurrence of asthma exacerbation, asthma control level, FeNO, and pulmonary functions.

\section{Methods}

\section{Subjects}

Patients with asthma who attended outpatient clinics at Kinki University Hospital, Keio University Hospital, Fukushima Medical University Hospital, Kagoshima University Medical and Dental Hospital, Teikyo University Hospital, Kurume University Hospital, and Shizuoka General Hospital for routine check-ups between August 2011 and May 2012were enrolled in this study. All patients satisfied the definition of asthma as defined by GINA [1]. Atopy was defined by positive specific IgE antibodies to at least one common inhalant allergen (CAP system; Pharmacia, Uppsala, Sweden). Inclusion criteria were as follows: (1) age over18 years; (2) ability to perform an adequate forced expiratory maneuver; (3) asthma duration more than 6 months; (4) receiving fixed-dose $\mathrm{FBC} 4.5 / 160 \mu \mathrm{g}^{2}$ inhalations bid for at least 3 months; (5) achievement of controlled asthma as defined by GINA (daytime symptoms, none or less than twice per week; limitation of activities, none; nocturnal symptoms/awakening, none; need for reliever/rescue treatment, none or less than twice per week; and lung function, normal) [1] for at least 3 months; and (6) an Asthma Control Questionnaire, 5-item version (ACQ5) score $\leq 0.75$. Patients were excluded from the study if they (1) were current smokers or had quit smoking within 1 month prior to the study; (2) had a smoking history more than 10 pack-years; (3) had chronic obstructive pulmonary disease, bronchiectasis, lung cancer, collagen vascular disease, pulmonary hypertension, old pulmonary tuberculosis, diffuse panbronchiolitis, or interstitial pneumonia; or (4) had a history of hospitalization within 1 year, or an emergency department visit or systemic corticosteroid treatment within 3 months due to asthma symptoms. Peak expiratory flow (PEF) monitoring was performed using a Mini wright peak flow meter (Clement Clarke Int. Ltd, Harlow, UK).

\section{Study design}

This was a prospective, multicenter, open-label, uncontrolled observational study. At visit 1, after 4 weeks of a run-in period, patients completed the ACQ5 and patients with a score $\leq 0.75$ were eligible for this study. FBC $4.5 / 160 \mu \mathrm{g}^{2}$ inhalations bid were stepped down to 1 inhalation bid and they attended the hospitals at weeks 4and 8 of the treatment period, as shown in Figure 1. The inhaler technique of each patient was checked by the involved doctors, nurses, or pharmacists not only during the run-in period but after stepping down the regimens. This study was conducted in accordance with the principles of the Declaration of Helsinki, this protocol was approved by each local ethics committee (UMIN000005406) and informed consent was obtained from all patients prior to the study.

\section{Measurements}

The primary endpoint was the occurrence of asthma exacerbation. Secondary endpoints, including ACQ5, FeNO, and pulmonary function tests, were measured in that order at weeks 0,4 , and 8 .

Asthma exacerbation: Asthma exacerbation was defined as hospitalization, emergency department visit, systemic corticosteroid treatment, or $>12$ puffs of short-acting $\beta 2$-agonist (SABA) use for 3 days due to asthma symptoms according to the recommendations with modifications [8].

ACQ5: The ACQ5 (Japanese version) consists of 5 items assessing nocturnal waking, morning symptoms, activity limitation, shortness of breath, and wheeze during the previous 7 days, excluding the frequency of SABA use and $\mathrm{FEV}_{1} \%$ predicted, each scored on a scale of $0-6$, where 0 represents good control and 6 represents poor control [9]. The overall score of the ACQ5 is the mean of the five responses. The cut-point for well-controlled asthma is $\leq 0.75$, and a value $\geq 1.50$ confirms not wellcontrolled asthma [10]. A 0.5 change in each score was considered a clinically meaningful difference [8].

FeNO: FeNO was measured by an online method using a handheld NO analyzer (NIOX MINO; Aerocrine AB, Solna, Sweden) according to the American Thoracic Society/European Respiratory Society recommendations [11]. Subjects emptied their lungs, inhaled deeply through the filter to total lung capacity, and then exhaled at

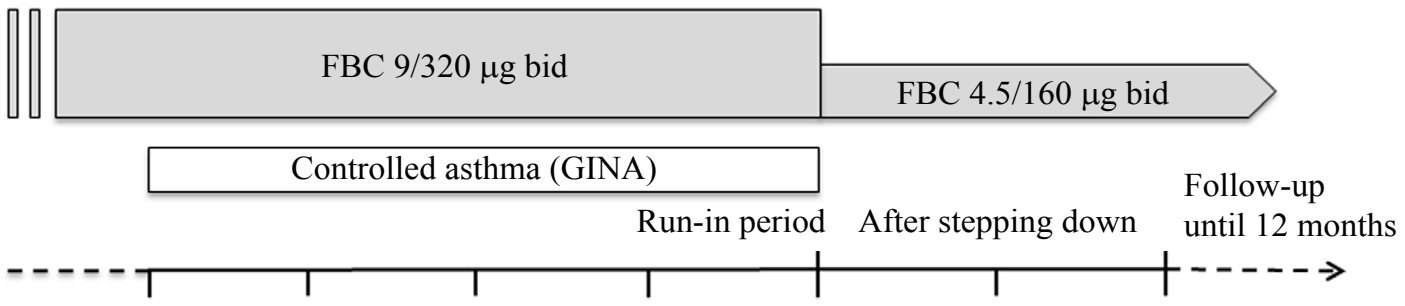

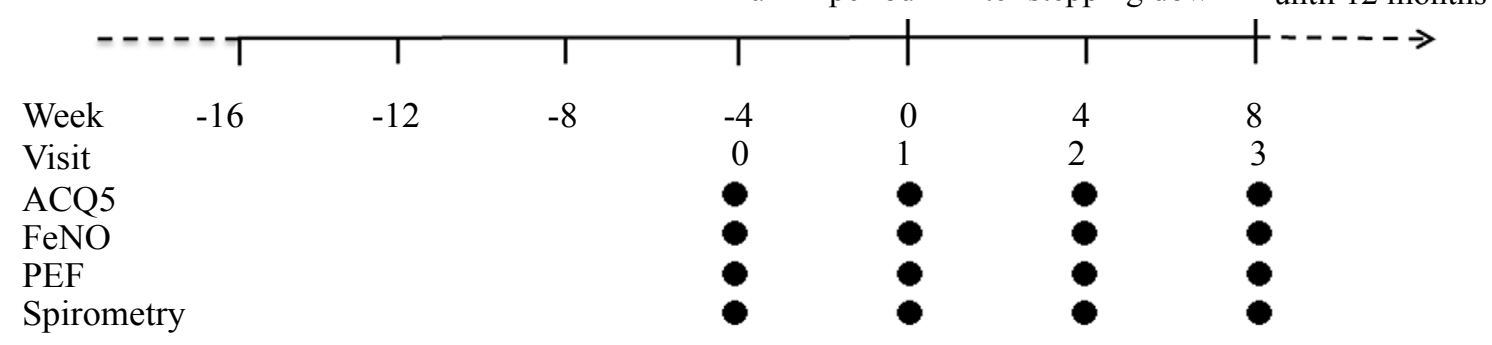

Figure 1: Study design

ACQ5: Asthma Control Questionnaire: 5-item version; FBC: Formoterol/Budesonide Combination; FeNO: Fractional Exhaled Nitric Oxide; GINA: Global Initiative for Asthma; PEF: Peak Expiratory Flow. 
a flow rate of $50 \mathrm{~mL} / \mathrm{s}$ (assisted by visual and auditory cues) for 10 seconds. Repeated and reproducible exhalations were performed to obtain at least 3 NO plateau values that agreed within $10 \%$ of each other. The average of 3 plateau values was recorded.

A previous study reported $36.8 \mathrm{ppb}$ as the upper limit of FeNO for healthy Japanese adults [12]. According to the cut-off levels of $37 \mathrm{ppb}$ at stepping down, patients were classified into 2 groups: high FeNO and low FeNO groups ( $\geq 37 \mathrm{ppb}$ versus $<37 \mathrm{ppb}$ ). 5.3.4. Pulmonary functions: $\mathrm{FVC}, \mathrm{FEV}_{1}$, forced expiratory flow at $50 \%$ of $\mathrm{FVC}\left(\mathrm{FEF}_{50 \%}\right)$, and $\mathrm{FEF}_{75 \%}$ were expressed as a percentage of predicted values according to the formula of the Japanese Respiratory Society [13] using an electrical spirometer, which was calibrated once a week, at each institution. The mean diurnal PEF variability and the lowest PEF expressed as a percentage of the highest PEF (Min\% Max $\mathrm{PEF}$ ) values [14] during the 1 -week period prior to each visit were calculated. Patients were requested not to use SABA for at least $6 \mathrm{~h}$ prior to the measurement.

\section{Long-term follow-up}

From 8 weeks until 12 months after stepping down, patients attended outpatient clinics at each institute for routine check-ups monthly, bimonthly, or trimonthly. The involved doctors were requested to maintain the reduced dose of FBC 4.5/160 $\mu \mathrm{g} 1$ inhalation bidas long as possible. Any measurements depended on each involved doctor. The occurrence of asthma exacerbation was assessed by medical records after 12 months.

\section{Statistical analysis}

All data are expressed as the medians with interquartile ranges. Comparisons between groups were made using the Kruskal-Wallis test or Wilcoxon signed-rank test. The chi-square or Fisher's exact test was used to test for significance in group differences. Comparisons between groups and between pre- and post-stepping down were performed by 2 -way repeated measures analysis of variance (ANOVA). A receiver- operator characteristic (ROC) curve was constructed to assess which level of FeNO would predict asthma exacerbation. StatView Version 5.0 (SAS Institute, Cary, NC) and R version 2.11.1 (The R Foundation for Statistical Computing, Vienna, Austria, 2010) were used for statistical calculations. A p value of $<0.05$ was considered significant, and all tests were 2-sided.GPower 3.1.9.2 (http://www.gpower.hhu.de/) was used for power calculations.

\section{Results}

Thirty-seven patients receiving a fixed-dose FBC $9 / 320 \mu \mathrm{g}$ bid and achieving controlled asthma (GINA) for at least 3 months and an ACQ5 score $\leq 0.75$ were enrolled. Based on the FeNO value at stepping down, patients were classified into 2 groups; 25 patients with $\mathrm{FeNO}<37$ $\mathrm{ppb}$ and 12 with FeNO $\geq 37 \mathrm{ppb}$ (Figure 2). The characteristics of the patients are shown in Table 1 . Patients with FeNO $\geq 37 \mathrm{ppb}$ had a significantly higher rate of coexisting allergic rhinitis and higher \%FVC values than those with $\mathrm{FeNO}<37 \mathrm{ppb}$. Allergic rhinitis was defined from interviews, medical records, or subjects own reporting. Within 8 weeks after stepping down to FBC $4.5 / 160 \mu \mathrm{g}$ bid, one of the patients with $\mathrm{FeNO}<37 \mathrm{ppb}$ developed asthma exacerbation, but none in those with $\mathrm{FeNO} \geq 37 \mathrm{ppb}$ (Figure 2).

The changes in ACQ5, pulmonary functions, and FeNO until 8 weeks after stepping down are shown in Table 2. Greater than 0.5 score of changes in ACQ5was observed in one patient with FeNO $<37$ $\mathrm{ppb}$ and 2 with $\mathrm{FeNO} \geq 37 \mathrm{ppb}$, respectively; however, there was no statistically significant change in ACQ5 and pulmonary functions in each group or any difference between the 2 groups by 2-way repeated measures ANOVA. FeNO in the patients with $\mathrm{FeNO}<37 \mathrm{ppb}$ increased significantly but not in those with $\mathrm{FeNO} \geq 37 \mathrm{ppb}$; however, there was no significance between the 2 groups by 2 -way repeated measures ANOVA.

In a long-term follow up until 12 months after stepping down, one patient with $\mathrm{FeNO}<37 \mathrm{ppb}$ and 4 with $\mathrm{FeNO} \geq 37 \mathrm{ppb}$ developed

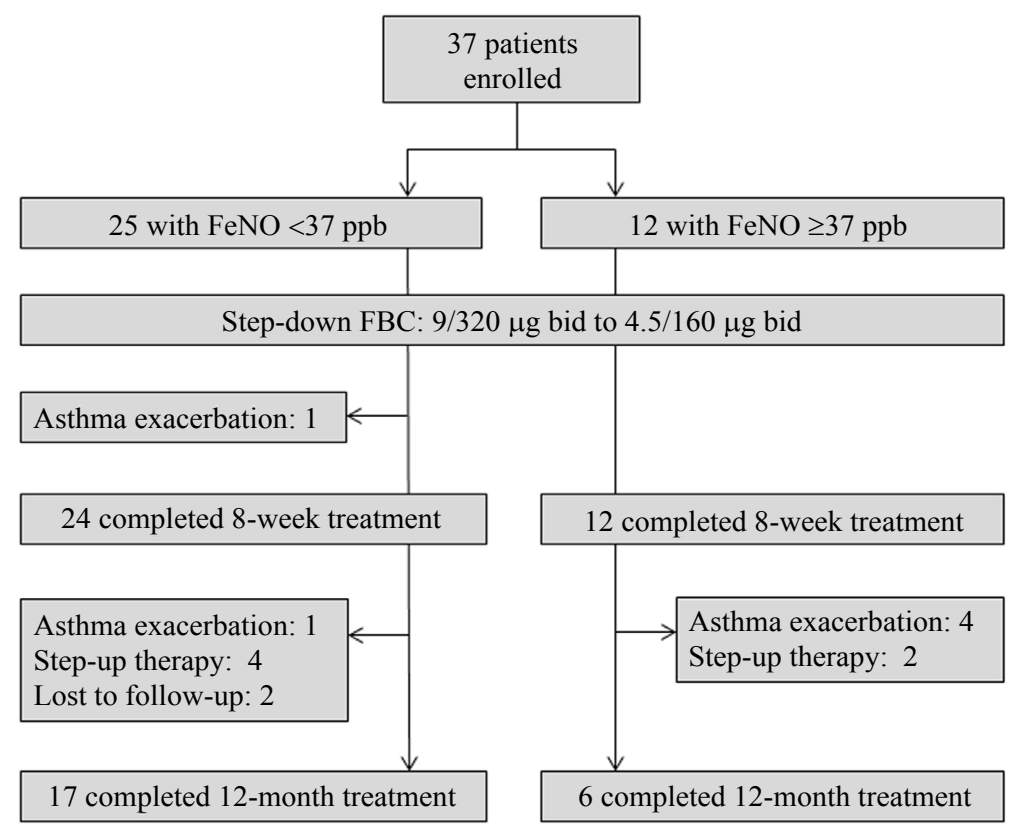

Figure 2: Flow of patient in study

FBC: Formoterol/Budesonide Combination; FeNO: Fractional Exhaled Nitric Oxide 
Citation: Shirai T, Kawayama T, Nagase H, Inoue H, Sato S, et al. (2014) Exhaled Nitric Oxide Measurement may Predict Asthma Exacerbation after Stepping down Formoterol/Budesonide Combination Therapy in Adult Asthma. J Allergy Ther 5: 173. doi:10.4172/2155-6121.1000173

Page 4 of 6

\begin{tabular}{|c|c|c|c|c|}
\hline & $\begin{array}{l}\text { All patients } \\
\quad(n=37)\end{array}$ & $\begin{array}{l}\text { Patients with FeNO<37 ppb } \\
\qquad(n=25)\end{array}$ & $\begin{array}{c}\text { Patients with FeNO } \\
\geq 37 \mathrm{ppb} \\
(\mathrm{n}=12)\end{array}$ & p-Value \\
\hline Age (years) & $61(49-69)$ & $61(39-68)$ & $65(53-70)$ & 0.3379 \\
\hline Gender, male/female & $15 / 22$ & $10 / 15$ & $5 / 7$ & 0.9230 \\
\hline Body mass index $\left(\mathrm{kg} / \mathrm{m}^{2}\right)$ & $23.0(19.4-25.4)$ & $23.4(19.4-26.1)$ & $21.9(19.7-24.6)$ & 0.6037 \\
\hline Atopic/nonatopic & $27 / 10$ & $18 / 7$ & $9 / 3$ & 0.8475 \\
\hline Non/ex-smokers & $29 / 8$ & $20 / 5$ & $9 / 3$ & 0.7295 \\
\hline Duration of asthma, years & $5.0(1.0-14.0)$ & $5.0(2.0-16.0)$ & $4.5(1.0-10.0)$ & 0.2032 \\
\hline Coexisting allergic rhinitis & 16 & 8 & $8^{*}$ & 0.0463 \\
\hline ACQ5, overall score & $0.0(0.0-0.2)$ & $0.0(0.0-0.2)$ & $0.0(0.0-0.1)$ & 0.5040 \\
\hline FVC, L & $3.14(2.58-3.70)$ & $3.02(2.43-3.44)$ & $3.47(2.67-4.05)$ & 0.2427 \\
\hline$\% F V C$ (\% predicted) & $106.2(88.6-113.5)$ & $97.9(88.0-109.6)$ & $115.8(104.6-134.2)$ & 0.0071 \\
\hline FEV1, L & $2.38(1.80-2.91)$ & $2.36(1.80-2.91)$ & $2.43(1.86-2.97)$ & 0.6731 \\
\hline \%FEV1 (\% predicted) & $89.4(80.6-108.2)$ & $88.2(80.6-105.1)$ & $106.2(79.1-112.0)$ & 0.2561 \\
\hline$\% \mathrm{FEF}_{50 \%}(\%$ predicted $)$ & $63.1(43.3-94.2)$ & $64.0(47.8-102.9)$ & $57.7(40.0-91.9)$ & 0.3636 \\
\hline$\% \mathrm{FEF}_{75 \%}(\%$ predicted $)$ & $45.5(29.0-76.6)$ & $46.5(34.8-81.1)$ & $37.4(25.8-62.5)$ & 0.1630 \\
\hline PEF variability, $\%$ & $3.3(2.0-4.8)$ & $3.3(2.0-5.7)$ & $2.7(1.8-4.6)$ & 0.6063 \\
\hline Min\%Max PEF, \% & $90.2(87.7-93.0)$ & $90.3(87.1-93.0)$ & $90.0(87.8-94.8)$ & 0.8539 \\
\hline FeNO, ppb & $28.0(18.5-38.5)$ & $20.0(18.0-28.0)$ & $51.5(40.8-57.5)$ & $<0.0001$ \\
\hline
\end{tabular}

Data are expressed as the medians (interquartile ranges).

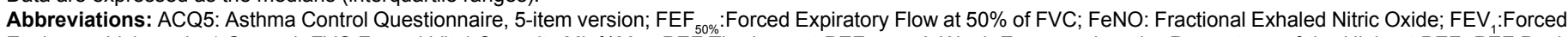
Expiratory Volume In 1 Second; FVC:Forced Vital Capacity;Min\%Max PEF:The Lowest PEF over A Week Expressed as the Percentage of the Highest PEF; PEF:Peak Expiratory Flow. ${ }^{*} p<0.05$ versus patients with FeNO $<37$ ppb.

Table 1: Characteristics of the study subjects at baseline.

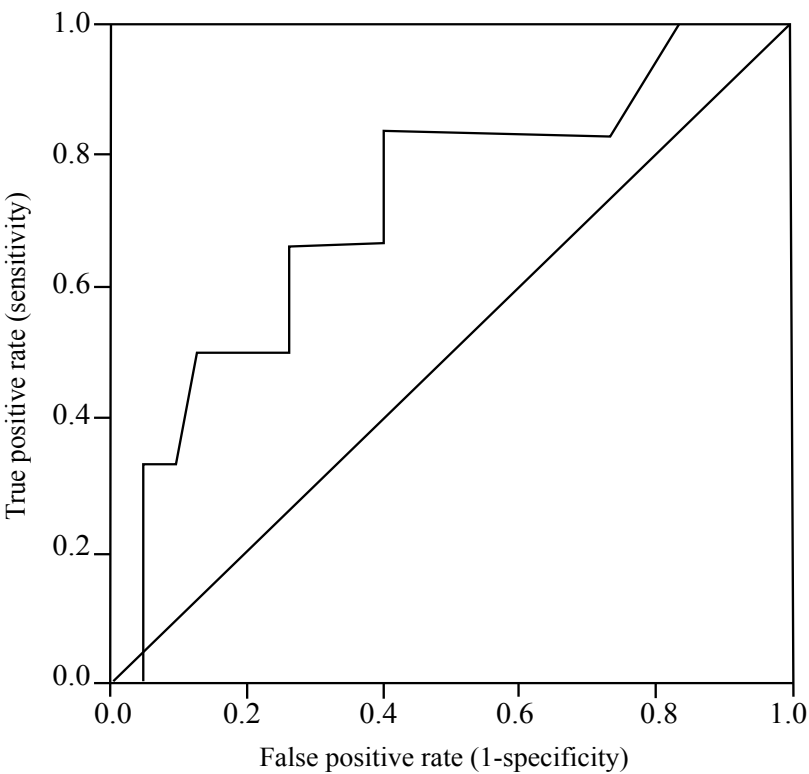

Figure 3: A receiver operator characteristic $(\mathrm{ROC})$ curve for FeNO. FeNO: Fractional Exhaled Nitric Oxide

asthma exacerbation (Figure 2). Therapy was stepped up again without exacerbation in 4 patients with $\mathrm{FeNO}<37 \mathrm{ppb}$ and 2 with $\mathrm{FeNO} \geq$ $37 \mathrm{ppb}$ by the involved doctors. The incidence of exacerbation was significantly higher in patients with $\mathrm{FeNO} \geq 37 \mathrm{ppb}$ than in those with $\mathrm{FeNO}<37 \mathrm{ppb}$ (odds ratio 11.33 , 95\% confidence interval 1.45 to 88.52 (Table 3).

The ROC curve is shown in Figure 3 and the area under the curve was 0.728 . The best cut point for predicting exacerbation was $27 \mathrm{ppb}$ (sensitivity, 0.833; and specificity, 0.609) and the second best was 36 ppb (sensitivity, 0.667; and specificity, 0.739).A re-analysis revealed that using the cut off value of $27 \mathrm{ppb}$, one patient with $\mathrm{FeNO}<27 \mathrm{ppb}$ and 4 with $\mathrm{FeNO} \geq 27 \mathrm{ppb}$ developed asthma exacerbation whereas 11 with $\mathrm{FeNO}<27 \mathrm{ppb}$ and 12 with $\mathrm{FeNO} \geq 27 \mathrm{ppb}$ did not, which was not statistically significant.

\section{Discussion}

We assessed whether FeNO measurement predicts asthma exacerbation after stepping down from fixed dose FBC 9/320 $\mu \mathrm{g}$ bid to $4.5 / 160 \mu \mathrm{g}$ bid. It was demonstrated that the incidence of exacerbation from 8 weeks until 12 months after stepping down was significantly higher in patients with $\mathrm{FeNO} \geq 37 \mathrm{ppb}$ than in those with $\mathrm{FeNO}<37$ ppb but that there was no difference in the incidence within 8 weeks between the 2 groups. These findings suggest that FeNO measurement at stepping down therapy may be useful for the long-term follow up of adult asthma as a predictor of asthma exacerbation.

Previous studies suggest that when asthma is controlled with ICS/ LABA combinations, the preferred approach to step-down therapy is to reduce the ICS dose by approximately $50 \%$ while continuing LABA, rather than discontinuing LABA alone [1]. Godard et al. [14] reported that in patients achieving asthma control with SFC $250 \mu \mathrm{g}$ bid, stepping down treatment with SFC $100 \mu \mathrm{g}$ bid was at least as effective on lung function and symptoms as continuing SFC 250, whereas fluticasone 250 $\mu \mathrm{g}$ bid was not. Since single inhaler maintenance and reliever therapy of FBC was not approved in Japan at the beginning of the present study, we investigated fixed-dose FBC step-down therapy.

Several reports have assessed the effect of stepping down treatment with ICS/LABA combinations, including FBC and SFC, on asthma control outcomes; however, very few have examined the usefulness of FeNO measurement in patients with well-controlled asthma. Recently, Hojo et al. [7] reported a study on step-down FBC therapy following the achievement of complete asthma control in terms of not only clinical symptoms but also eosinophilic airway inflammation. After receiving FBC $9 / 320 \mu \mathrm{g}$ bid for an average of 3 months, treatment was stepped down to $4.5 / 160 \mu$ g bidif FeNO $\leq 28 \mathrm{ppb}$ plus ACT score $\geq 22$ was met as a criterion. They found that the number of SABA use and moderate to severe exacerbation were comparable before and 48 weeks 


\begin{tabular}{|c|c|c|c|c|c|c|c|}
\hline & \multicolumn{3}{|c|}{ Patients with FeNO<37 ppb (n=25) } & \multicolumn{3}{|c|}{ Patients with FeNO $\geq 37$ ppb (n=12) } & \multirow{2}{*}{$\begin{array}{l}\text { ANOVA } \\
\text { p-Value }\end{array}$} \\
\hline & OW & $8 W$ & p-Value & oW & $8 W$ & p-Value & \\
\hline ACQ5, overall score & $0.0(0.0-0.2)$ & $0.0(0.0-0.2)$ & 0.2341 & $0.0(0.0-0.1)$ & $0.0(0.0-0.0)$ & 1.0000 & 0.7678 \\
\hline FVC, L & $3.02(2.43-3.44)$ & $3.12(2.45-3.58)$ & 0.6271 & $3.47(2.67-4.05)$ & $3.35(2.50-3.87)$ & 0.4769 & 0.6890 \\
\hline$\% F V C, \%$ & $97.9(88.0-109.6)$ & $98.1(86.7-109.8)$ & 0.5111 & $115.8(104.6-134.2)$ & $114.6(97.1-132.4)$ & 0.4236 & 0.7565 \\
\hline $\mathrm{FEV}_{1}, \mathrm{~L}$ & $2.36(1.80-2.91)$ & $2.29(1.72-2.79)$ & 0.0591 & $2.43(1.86-2.97)$ & $2.15(1.78-3.03)$ & 0.4145 & 0.8900 \\
\hline \%FEV1 (\% predicted) & $88.2(80.6-105.1)$ & $85.9(76.9-104.8)$ & 0.0593 & $106.2(79.1-112.0)$ & $101.9(72.0-111.0)$ & 0.3329 & 0.7697 \\
\hline$\% \mathrm{FEF}_{50 \%}, \%$ & $64.0(47.8-102.9)$ & $63.3(46.1-98.0)$ & 0.1137 & $57.7(40.0-91.9)$ & $49.8(31.4-91.9)$ & 0.2477 & 0.8567 \\
\hline$\% \mathrm{FEF}_{75 \%}, \%$ & $46.5(34.8-81.1)$ & $44.3(25.7-75.5)$ & 0.0765 & $37.4(25.8-62.5)$ & $25.5(18.8-69.5)$ & 0.2845 & 0.8266 \\
\hline PEF variability, \% & $3.3(2.0-5.7)$ & $3.0(2.0-4.7)$ & 0.1440 & $2.7(1.8-4.6)$ & $2.9(1.4-5.9)$ & 0.2026 & 0.8770 \\
\hline Min\%Max PEF, \% & $90.3(87.1-93.0)$ & $91.0(86.1-93.0)$ & 0.9405 & $90.0(87.8-94.8)$ & $91.5(86.1-95.6)$ & 0.4413 & 0.2957 \\
\hline FeNO, ppb & $20.0(18.0-28.0)$ & $25.5(18.8-34.5)$ & 0.0495 & $51.5(40.8-57.5)$ & $67.0(33.0-76.0)$ & 0.2298 & 0.7565 \\
\hline
\end{tabular}

Data are expressed as the medians (interquartile ranges).

Abbreviations: ACQ5: Asthma Control Questionnaire, 5-item version; ANOVA:Analysis Of Variance; FBC:Formoterol/Budenonide Combination; FEF50\%:Forced Expiratory Flow at $50 \%$ of FVC; FeNO:Fractional Exhaled Nitric Oxide;FEV1:Forced Expiratory Volume in 1 Second; FVC:Forced Vital Capacity; Min\%Max PEF:The Lowest PEF Over a Week Expressed as the Percentage of the Highest PEF; PEF:Peak Expiratory Flow. ${ }^{*} p<0.05$ versus 0 W.

Table 2: Changes in ACQ5, pulmonary functions, and FeNOuntil 8 weeks after stepping down.

\begin{tabular}{|l|l|c|c|}
\hline & & \multicolumn{2}{|c|}{ FeNO at baseline (visit 1) } \\
\hline \multirow{2}{*}{ Asthma exacerbation } & & $<37 \mathrm{ppb}$ & $\mathbf{3} \mathbf{3 7} \mathbf{~ p p b}$ \\
\cline { 2 - 4 } & Did not occur & 17 & 6 \\
\cline { 2 - 4 } & Did occur & 1 & 4 \\
\hline
\end{tabular}

Data were evaluated by Fisher's exact test $(p=0.0410)$. The odds ratio for the occurrence of asthma exacerbation was 11.33 (95\% confidence interval 1.45 to 88.52). Abbreviations: FeNO:Fractional Exhaled Nitric Oxide.

Table 3: Association between FeNO level at baseline and asthma exacerbation from 8 weeks until 12 months after stepping down.

after stepping down in 51 patients. In the present study, we found that $\mathrm{FeNO}<37 \mathrm{ppb}$ was a predictor of relatively safe step-down $\mathrm{FBC}$ therapy in a long-term follow-up of 37 patients achieving controlled asthma (GINA) for at least 3 months and an ACQ5 score $\leq 0.75$.There are several differences in the study design between Hojo et al. and the present study, including a slight shorter duration of asthma control prior to stepping down, more strict FeNO $(\leq 28 \mathrm{ppb})$ as a criterion, and comparing the incidence of exacerbation before and after stepping down in their study; however, these two studies show that complete control of asthma, not only clinical symptoms but also eosinophilic airway inflammation, is essential for successful step-down treatment. Also, we clarified that the future risks associated with step-down treatment may be related to the control of underlying inflammation activity, as reflected in biomarker levels.

We classified patients into high and low FeNO groups according to the cut-off of $37 \mathrm{ppb}$ at baseline, which was higher than $25 \mathrm{ppb}$, the level of less likelihood of eosinophilic inflammation recommended by the clinical practice guidelines [3]. This was because 16 of 37 patients (43\%) had allergic rhinitis, a factor associated with higher FeNO; however, the mean value was $22.2 \mathrm{ppb}$ in the low FeNO group while the mean baseline value of $55.5 \mathrm{ppb}$ in the high FeNO group corresponded to the level of eosinophilic inflammation indicated by the guidelines (3). Considering this and the results of ROC analysis, the cut-off was appropriate in the present study.

The occurrence of exacerbation just after stepping down means the failure of stepping down itself and the need to step up again. If there is no exacerbation in this period, stepping down therapy is successful in any way. The next problem is whether asthma control will be maintained in the presence of factors that precipitate exacerbation, including seasons, weather, temperature, and infections. That's the reason why we assessed within 8 weeks and from 8 weeks until 12 months separately.
FeNO measurement predicted asthma exacerbation not within 8 weeks but from 8 weeks until 12 months after step-down FBC therapy in the present study. A possible explanation may be that the effect of underlying eosinophilic airway inflammation on future exacerbation takes a certain period of time. Previous studies $[7,15,16]$ and the present study showed that FeNO increased gradually after step-down treatment. Future studies will clarify whether adjustment of therapy based on FeNO measurement can prevent or when to measure FeNO to prevent asthma exacerbation.

The involved doctors were requested to maintain the reduced dose of FBC 4.5/160 $\mu \mathrm{g}$ inhalation bid as long as possible; however, in some patients therapy was stepped up to respond to the patients' request. Actually, exacerbation did not occur but the patients preferred the initial dose before stepping down due to increased symptoms. It is true that this may bias the exacerbation results, but we excluded these patients from the analysis.

Regular FENO measurements rather than a single baseline would have clinical importance in predicting exacerbations; however, FeNO measuring devices are very expensive and not widely and frequently used in clinical practice. We believe that a single FeNO measurement, at least partly, gives us useful information on exacerbation.

The major limitation of the present study is a small sample size. Due to several circumstances, we could not collect more patients. The statistical power of the present study was $57.1 \%$. Further studies with more patients are needed to clarify the usefulness of FeNO measurements in stepping down therapy.

In conclusion, higher FeNO predicts asthma exacerbation from 8 weeks until 12 months, but not within 8 weeks, after stepping down from fixed-dose FBC $9 / 320 \mu \mathrm{g}$ bid to $4.5 / 160 \mu \mathrm{g}$ bid. FeNO $<37 \mathrm{ppb}$ may be a predictor of relatively safe step-down therapy of adult patients achieving well-controlled asthma.

\section{Acknowledgment}

We thank Tomoyoshi Tsuchiya, MD, PhD (Shizuoka City Shimizu Hospital, Department of Respiratory Medicine) for his assistance with statistical analysis.

\section{Funding}

This study was supported by a research grant from The Waksman Foundation of Japan Inc.

\section{References}

1. Global Initiative for Asthma. Global Strategy for Asthma Management and Prevention. 
Citation: Shirai T, Kawayama T, Nagase H, Inoue H, Sato S, et al. (2014) Exhaled Nitric Oxide Measurement may Predict Asthma Exacerbation after Stepping down Formoterol/Budesonide Combination Therapy in Adult Asthma. J Allergy Ther 5: 173. doi:10.4172/2155-6121.1000173

Page 6 of 6

2. Reddel HK, Taylor DR, Bateman ED, Boulet LP, Boushey HA, et al. (2009) An official American Thoracic Society/European Respiratory Society statement: asthma control and exacerbations: standardizing endpoints for clinical asthma trials and clinical practice. Am J Respir Crit Care Med 180: 59-99.

3. Dweik RA, Boggs PB, Erzurum SC, Irvin CG, Leigh MW, et al. (2011) An official ATS clinical practice guideline: interpretation of exhaled nitric oxide levels (FENO) for clinical applications. Am J Respir Crit Care Med 184: 602-615.

4. Shaw DE, Berry MA, Thomas M, Green RH, Brightling CE, et al. (2007) The use of exhaled nitric oxide to guide asthma management: a randomized controlled trial. Am J Respir Crit Care Med 176: 231-237.

5. Szefler SJ, Mitchell H, Sorkness CA, George PJ, O'Connor GT, et al. (2008) Management of asthma based on exhaled nitric oxide in addition to guidelinebased treatment for inner-city adolescents and young adults: a randomised controlled trial. Lancet 372: 1065-1072.

6. Gelb AF, Flynn Taylor C, Shinar CM, Gutierrez C, Zamel N (2006) Role of spirometry and exhaled nitric oxide to predict exacerbations in treated asthmatics. Chest 129: 1492-1499.

7. Hojo M, Mizutani T, likura M, Hirano S, Kobayashi N, et al. (2013) Asthma control can be maintained after fixed-dose, budesonide/ formoterol combination inhaler therapy is stepped down from medium to low dose. Allergol Int 62: 9198.

8. Fuhlbrigge A, Peden D, Apter AJ, Boushey HA, Camargo CA Jr, et al. (2012) Asthma outcomes: exacerbations. J Allergy Clin Immunol 129: S34-48.

9. Juniper EF, Svensson K, Mörk AC, Ståhl E (2005) Measurement properties and interpretation of three shortened versions of the asthma control questionnaire. Respir Med 99: 553-558.

10. Juniper EF, Bousquet J, Abetz L, Bateman ED; GOAL Committee (2006) Identifying 'well-controlled' and 'not well-controlled' asthma using the Asthma Control Questionnaire. Respir Med 100: 616-621.

11. American Thoracic Society; European Respiratory Society (2005) ATS/ERS recommendations for standardized procedures for the online and offline measurement of exhaled lower respiratory nitric oxide and nasal oxide, 2005 Am J Respir Crit Care Med 171: 912-930.

12. Matsunaga K, Hirano T, Kawayama T, Tsuburai T, Nagase H, et al. (2010) Reference ranges for exhaled nitric oxide fraction in healthy Japanese adult population. Allergol Int 59: 363-367.

13. The Committee of Pulmonary Physiology, Japanese Respiratory Society (2004) Guidelines for Pulmonary Function Tests. Spirometry, flow-volume curve, diffusing capacity of the lung. Tokyo: The Japanese Respiratory Society

14. Reddel HK, Salome CM, Peat JK, Woolcock AJ (1995) Which index of peak expiratory flow is most useful in the management of stable asthma? Am J Respir Crit Care Med 151: 1320-1325.

15. Godard P, Greillier P, Pigearias B, Nachbaur G, Desfougeres JL, et al. (2008) Maintaining asthma control in persistent asthma: comparison of three strategies in a 6-month double-blind randomised study. Respir Med 102: 1124-1131.

16. Obase Y, Ikeda M, Kurose K, Abe M, Shimizu H, et al. (2013) Step-down of budesonide/formoterol in early stages of asthma treatment leads to insufficient anti-inflammatory effect. J Asthma 50: 718-721. 